\title{
Transcatheter closure of atrial septal defects using the Cardio-Seal implant
}

\author{
C A C Pedra, J Pihkala, K-J Lee, C Boutin, D G Nykanen, P R McLaughlin, \\ D A Harrison, R M Freedom, L Benson
}

\begin{abstract}
Objective-To review the outcomes of transcatheter closure of atrial septal defects using the Cardio-Seal implant.

Design-A prospective interventional study.

Setting-Tertiary referral centre.

Patients-The first 50 patients (median age 9.7 years) who underwent attempted percutaneous occlusion.

Interventions-Procedures were done under general anaesthesia and transoesophageal guidance between December 1996 and July 1998.

Main outcome measures-Success of deployment, complications, and assessment of right ventricular end diastolic diameter, septal wall motion, and occlusion status by echocardiography. Results-The median balloon stretched diameter was $14 \mathrm{~mm}$. Multiple atrial septal defects were present in 11 patients $(22 \%)$ and a deficient atrial $\mathrm{rim}(<4 \mathrm{~mm})$ in $19(38 \%)$. In four patients $(8 \%)$, a second device was implanted after removal of an initially malpositioned first implant. There were no significant immediate complications. All patients except one were discharged within 24 hours. At the latest follow up (mean 9.9 months) a small shunt was present in 23 patients $(46 \%)$, although right ventricular end diastolic dimensions (mean (SD)) corrected for age decreased from 137 (29)\% to 105 (17)\% of normal, and septal motion abnormalities normalised in all but one patient. No predictors for a residual shunt were identified. Supporting arm fractures were detected in seven patients (14\%) and protrusion of one arm through the defect in $16(32 \%)$, the latter being more common in those with smaller anterosuperior rims. No untoward effects resulted from arm fractures or protrusion. There were no complications during follow up, although five patients $(10 \%)$ experienced transient headaches.

Conclusions-The implantation of the Cardio-Seal device corrects the haemodynamic disturbances secondary to the right ventricular volume overload, with good early outcome.

(Heart 2000;84:320-326)
\end{abstract}

Keywords: congenital heart disease; interventional catheterisation; atrial septal defects

Transcatheter closure of the isolated secundum atrial septal defect has evolved over the last 30 years, with refinements in technology giving birth to a variety of devices. ${ }^{1-6}$ The Cardio-Seal occlusion device (Nitinol Medical Technologies Inc, Boston, Massachusetts, USA) is one such device. It was re-engineered from the former Bard Clamshell septal occluder (Bard Inc, Billerica, Massachusetts, USA), ${ }^{6}$ a double umbrella design that underwent multicentre clinical trials in both the USA and Canada between 1989 and 1991 with very favourable initial clinical results. However, a high rate of supporting arm fractures was noted in follow up, prompting discontinuation of elective implantations. $^{78}$ The device was redesigned and new trials were begun in late $1996 .{ }^{6}$ This study describes the preliminary results from a single institution with this new occlusion system.

Medicine, Division of Cardiology, Toronto General Hospital

P R McLaughlin

D A Harrison

Correspondence to: Dr Benson

email:

benson@sickkids.on.ca

Accepted 10 April 2000

\section{Methods}

PATIENT POPULATION

Between December 1996 and July 1998, 50 patients (12 male and 38 female) from 3.5 to 71 years of age (mean 15.9; median 9.7) underwent attempted percutaneous transvenous atrial septal defect occlusion using the Cardio-Seal implant. Weight ranged from
11.4-104 kg (mean 37.4; median 32.2), and body surface area ranged from $0.6-2.3 \mathrm{~m}^{2}$ (mean 1.18; median 1.13). Associated defects (all mild) included pulmonary valve stenosis in two patients, left ventricular outflow obstruction in one, and right ventricular hypoplasia with a small ventricular septal defect in another.

All parents or patients (when applicable) agreed to participate in the clinical trial approved by the Ministry of Health (Health Protective Branch, Ottawa, Canada). Informed consent was obtained from all patients or parents following guidelines of the human subject protection committee of the Hospital for Sick Children, University of Toronto, Canada.

STUDY PROTOCOL

Patients were screened by transthoracic cross sectional colour Doppler echocardiography using multiple standard views. Inclusion criteria included:
- the presence of an ostium secundum atrial septal defect;

- left to right shunting across the septum;

- maximum atrial septal defect diameter of $20 \mathrm{~mm}$;

- a dilated right ventricle with evidence of volume overload; 
- a safe distance (usually $4-5 \mathrm{~mm}$ ) from the margins of the defect to intracardiac structures (atrioventricular valves, superior caval vein, upper right pulmonary vein, and coronary sinus);

- an adequate rim of tissue $(>4 \mathrm{~mm})$ present around at least $75 \%$ of the defect circumference;

- size of the patient large enough to accommodate an 11 French sheath (usually more than 8-10 kg or over two years of age).

Exceptions were made in the case of two female patients (19 and 30 years old) with a diagnosis of transient ischaemic attacks presumed to be caused by paradoxical embolisation. Contrast transoesophageal echocardiography (TOE) showed a patent oval foramen shunting right to left with a small aneurysm of the atrial septum in one of these, and a small secundum atrial septal defect with bidirectional shunting but no right ventricular volume overload in the other. Both patients had histories of recurrent transient episodes of hemiparesis and slurred speech, and were on warfarin at the time of catheterisation, with poor compliance.

All patients were taken to the cardiac catheterisation laboratory and evaluated by TOE under general anaesthesia before catheterisation. TOE reassessed the size of the defect and the surrounding rims, the length of the atrial septum, and pulmonary venous connections. Patients who did not fulfil the criteria described above were referred for surgical intervention. The patient with mild right ventricular hypoplasia and a small ventricular septal defect had test occlusion of the atrial septal defect before attempted device closure, with no change in right atrial pressures or decrease in cardiac output.

DEVICE AND DELIVERY SYSTEM

The Cardio-Seal device consists of two self expanding umbrellas that are attached to the atrial septum by spring tension after implantation. Four metal arms made from the metal alloy MP35N radiate from the centre of the device, supporting each umbrella. These arms are attached to each other in the centre and covered by sewn square Dacron patches. There are two coil joints or hinges in each arm designed to relieve the stresses placed upon the implant during the cardiac cycle, improving resistance to fatigue fractures when tested in vitro. $^{6}$ In addition, the spring back mechanism - which allows the umbrella frame to assume its original shape after being deformed during delivery, fixing the device to the atrial septum-was also improved in the new design. The device comes in five nominal sizes that correspond to the diagonal length of each umbrella: $17,23,28,33$, and $40 \mathrm{~mm}$. It is loaded into the distal pod of the delivery catheter with the aid of a plastic loader.

PROCEDURE AND TECHNIQUE OF IMPLANTATION All procedures were performed under general endotracheal anaesthesia with TOE guided positioning of the device. ${ }^{9}$ After percutaneous entry of the femoral vein, a complete haemody- namic evaluation was performed. To delineate the anatomy of the atrial septal defect, an angiogram was obtained in the right upper pulmonary vein in a hepatoclavicular projection ( $30^{\circ}$ cranial and $30^{\circ}$ left anterior oblique). Subsequently, an end hole catheter was passed through the defect and an exchange $260 \mathrm{~cm}$ extra stiff Amplatz guide wire (Cook, Bloomington, Indiana, USA) placed in the left upper pulmonary vein. Over this wire, a $20 \mathrm{~mm}$ occlusion balloon catheter (Meditech, Watertown, Massachusetts, USA) was advanced into the left atrium. The balloon catheter was inflated with an equal mixture of contrast and saline and pulled towards the atrial septum under fluoroscopic and TOE guidance. After confirmation of occlusion of the defect by the balloon, the presence of any additional defects within the atrial septum was sought by colour Doppler. If any were found, their size, location, and distance from the central defect were defined. A cineangiogram was obtained of the balloon being pulled gently and steadily against the septum causing its deformation as it passed through the defect. This deformation-the stretched diameter - was then measured, using the catheter as magnification correction. The device diameter was selected to be at least twice the size of the stretched diameter of the defect. In order to avoid implanting an oversized device, particularly in small patients with large defects, the total diameter of the atrial septum was also measured and compared with the size of the selected device. When additional defects were present, the selected device was implanted only if it was felt that it would cover all the defects.

After sizing the defect, a long sheath and dilator (11 French, Cook) were advanced over the guide wire into the left atrium. To avoid air entry, a continuous infusion of heparinised saline was maintained through a side arm attached to the dilator of the long sheath, starting before the system was introduced through the skin. After crossing the defect, the sheath was advanced over the dilator, and the wire and dilator were carefully removed. The chosen device was loaded into the delivery catheter and advanced through the long sheath into the inferior caval vein and to the right atrial junction. Pushing the delivery wire on the delivery catheter, the device was advanced out of the distal pod into the sheath until the distal arms were fully opened in the left atrial cavity. Care was taken to avoid entrapment of the device within the left atrial appendage or pulmonary veins. Under TOE guidance, the entire system was pulled back gently until the left atrial umbrella approached or slightly touched the left atrial side of the septum. This was generally in the region of the anterosuperior portion of the septum, and care was taken not to pull an arm through to the right atrium. Indeed, to achieve this position, the centre of the implant tended to be on the left atrial side of the defect before the right atrial arms were opened (fig 1). If the orientation of the device was perpendicular to the plane of the atrial septum, the device could protrude through the defect. If this occurred attempts were made to 

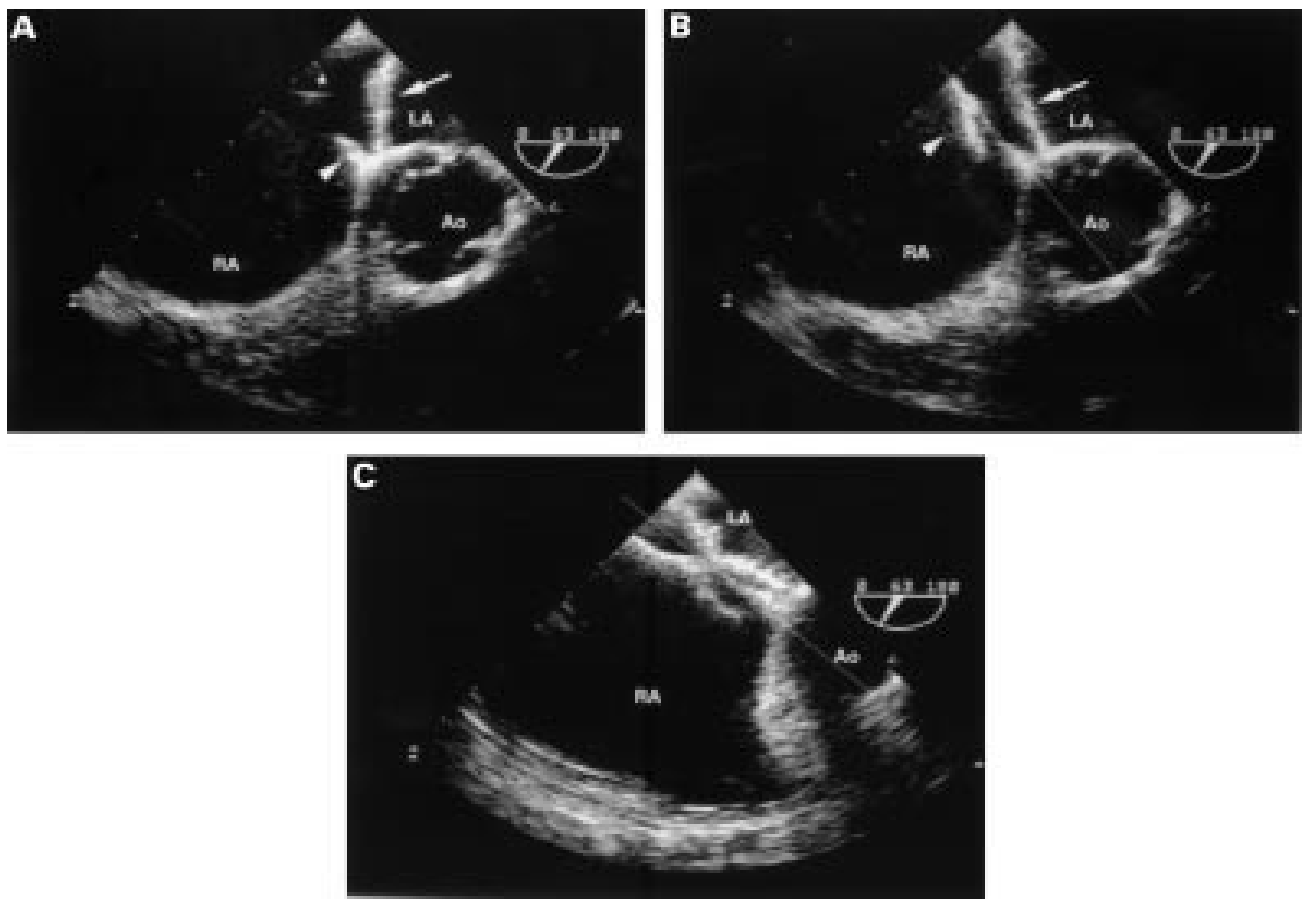

Figure 1 Transoesophageal echocardiograms obtained during implantation; short axis view. (A) The left atrial umbrella, seen in profile (arrow), is pulled towards the atrial septum, approaching the anterosuperior septal rim (arrow head). Note that the centre of the implant is on the left side of the septum before the right atrial arms are opened. Delivery wire marked with asterisk. (B) The right atrial umbrella (arrow head) is opened with the device slightly off plane of the interatrial septum (dotted line). (C) After release, the device aligns to the plane of the interatrial septum (dotted line). Ao, aorta; LA, left atrium; $R A$, right atrium.

bring it into a more parallel position by rotation (sheath and delivery catheter) or by recapturing it in the sheath (refolding by retracting the delivery wire), with redeployment of the left atrial disc.

When a satisfactory position was obtained, the sheath was further retracted over the delivery catheter, opening the proximal disc within the right atrium. To avoid distortion of the atrial septum by the relatively stiff attachment of the device to the wire, the sheath was fully retracted to the inferior caval vein. While connected to the delivery wire, proper positioning of the device was confirmed by TOE. If the device was in a stable location the release mechanism was activated and the device detached. TOE was again employed to confirm the final position of the device and evaluate the presence, location, and size of possible residual shunting. Three dimensional echocardiographic reconstruction was obtained in 24 patients immediately after the implantation, as part of another approved protocol. ${ }^{10}$ Cefazolin $(40 \mathrm{mg} / \mathrm{kg}$, maximum dose $1 \mathrm{~g}$ ) and heparin sulphate (150 IU/kg, maximum $5000 \mathrm{IU})$ were given immediately before device implantation. After removal of the sheath, haemostasis was achieved by local pressure, and the patient was allowed to waken in the catheterisation laboratory. A second dose of antibiotics was given after eight hours and the patient was discharged home the following day on low dose of aspirin (3-5 mg/kg daily; maximum $325 \mathrm{mg}$ ) for six months.

FOLLOW UP STUDIES

A chest radiograph and a transthoracic colour Doppler echocardiogram were obtained before discharge. Clinical assessment with chest $x$ ray and ECG was performed at one, six, and 12 months of follow up. Transthoracic echocardiography was repeated six and 12 months after the procedure. The location and size of residual shunting were classified on echocardiography according to previously published protocols. ${ }^{9}$ Right ventricular end diastolic dimensions were measured in all patients and normalised against predicted values for age. ${ }^{11}$ A fluoroscopic evaluation was also performed at the six month review. Arm fractures were identified on chest $x$ ray or fluoroscopy. A 24 hour ambulatory ECG was obtained if there was any evidence of arrhythmias on standard ECG or new symptoms suggesting rhythm disturbances.

\section{STATISTICAL ANALYSIS}

All values are expressed as mean (SD) or median and range, as applicable. A paired Student's $t$ test was used to compare changes in right ventricular size, atrial septal defect diameters (stretched and on TOE), and device diameter to stretched diameter ratios. Multiple logistic regression was performed to identify factors predictive of a residual leak. A $\chi^{2}$ test was used to compare changes in the septal motion, and a Fischer exact test was used to evaluate the association between protrusion of the device, residual shunts, and partial deficiency in septal rims. The level of significance was set at $\mathrm{p}<0.05$. The statistical analysis was performed using the SigmaStat version 2.0 (1995) statistical software (Jandel Corporation, San Rafael, California, USA). 
Results

According to the selection criteria by transthoracic echocardiography, 63 patients were taken to the catheterisation laboratory. Subsequent TOE showed that 13 patients were unsuitable for catheter closure for various reasons. These included a deficient rim ( $<4 \mathrm{~mm}$ ) surrounding more than $50 \%$ of the circumference of the defect (five patients), the size of defect $>18 \mathrm{~mm}$ (four patients), and, in one patient each, a large defect in a patient thought to be too small to accommodate the device, the presence of a sinus venosus defect with partial anomalous pulmonary venous connection, the presence of an inferior caval vein defect, and a small defect with no right ventricular volume overload. Transcatheter closure of the defect was carried out successfully in the remaining 50 patients.

The mean (SD) defect diameter measured by TOE was 11.9 (2.9) $\mathrm{mm}$ (range 7-18 mm; median $12 \mathrm{~mm}$ ) and was significantly smaller than the mean stretched diameter of 13.7 (3.2) $\mathrm{mm}$ (range 7.5-20 mm; median $14 \mathrm{~mm}$ ) $(\mathrm{p}<0.0001)$. Two defects were found in 10 of the 50 patients $(20 \%)$ and a fenestrated septum in one $(2 \%)$. A deficient $\operatorname{rim}(<4 \mathrm{~mm})$ was present in the anterosuperior location in 17 patients $(34 \%)$ and in the posteroinferior rim in two $(4 \%)$. The measured $\mathrm{Qp} / \mathrm{Qs}$ (ratio of pulmonary to systolic blood flow) was 1.9:1 (0.7) (range 1-4), and right ventricular and pulmonary artery pressures were normal in all patients. Fluoroscopy time ranged from 7-32 minutes (mean 15.5 (5.4) minutes). Four sizes of device were used: $23 \mathrm{~mm}(\mathrm{n}=4,8 \%)$, $28 \mathrm{~mm}(\mathrm{n}=13,26 \%), 33 \mathrm{~mm}(\mathrm{n}=16,32 \%)$, $40 \mathrm{~mm}(\mathrm{n}=17,34 \%)$. The ratio of device diameter to the stretched diameter of the defect was 2.5 (0.4) (range 1.9-3.6).

In four of the 50 patients $(8 \%)$, the initial device was refolded into the sheath while still attached to the delivery wire after deployment of both umbrellas. In one patient each, the proximal inferior arm caught the Eustachian valve during closure of the right atrial disc; a distal umbrella protruded from left to right atrium; the device was too large for the length of the atrial septum, or the device failed to release properly after adequate positioning. A second device was implanted successfully in all these instances.

Minor immediate complications occurred in three patients $(6 \%)$ : one short episode of supraventricular tachycardia requiring adenosine treatment; one episode of transient Mobitz I atrioventricular block requiring atropine; and one episode of transient ST elevation with spontaneous recovery. An immediate TOE (less than 20 minutes after the implantation) showed a residual leak in 30 patients $(60 \%)$. No flow disturbance was observed in the superior or inferior caval vein, the coronary sinus, or the right upper pulmonary vein. In no instance did the device impinge on the mitral or tricuspid valves resulting in regurgitation. There were no device embolisations, deaths, vascular complications, or blood transfusions. All patients except one were discharged home on the following day. At discharge, 30/50 patients
$(60 \%)$ had residual shunting assessed by transthoracic echocardiography.

Mean follow up was 9.9 (3.2) months (range 6-12 months). At the latest follow up, persistent shunting was identified in 23 patients $(46 \%)$, estimated at $<2 \mathrm{~mm}$ in diameter in $13(26 \%)$, between $2-4 \mathrm{~mm}$ in four ( $8 \%$ ), and $>4 \mathrm{~mm}$ in six $(12 \%)$. The length of follow up did not differ between patients with or without residual leaks. The shunt occurred around the device in 18 patients, through the device in four, and both through and around the device in one. No risk factors - including the diameter of atrial septal defect on TOE, the stretched diameter of the defect, the size of the device, deficiency of the rim, or the presence of multiple defects - were predictive of residual shunting. The two patients who had presumed stroke from right to left shunting had small $(<2 \mathrm{~mm})$ left to right shunts on transthoracic echocardiography 12 months after device implantation, but no right to left shunting detected on a contrast bubble study. Both patients complained of episodes of visual disturbance and blurred vision within the first month after implantation. No cerebral ischaemic episodes occurred at follow up, and repeat brain computed tomography revealed no new abnormalities.

The echocardiographic right ventricular end diastolic dimension corrected for age was 137 (29)\% of predicted before implantation, and $105(17) \%$ at latest follow up $(p<0.0001)$. Increased right ventricular end diastolic dimensions persisted in 23/50 patients (46\%). Patients with complete closure had a reduction in right ventricular size of 29 (13)\% $(\mathrm{p}<0.0001)$, which was greater than in patients with residual leaks (18 (15)\%) $(p=0.01)$. The length of follow up did not differ between groups. Septal motion abnormalities were present in 48 of the patients (96\%) before implantation and returned to normal in all but one $(2 \%)$, who had a $1 \mathrm{~mm}$ residual leak associated with flattened septal motion $(p<0.001)$. Of the six patients with leaks measuring $>4 \mathrm{~mm}$ by colour Doppler, four had normal right ventricular dimensions and septal wall motion.

Fractures of the device arm(s) were detected in seven patients $(14 \%)$, of whom four had a $33 \mathrm{~mm}$ device (one with multiple fractures) and three had a $40 \mathrm{~mm}$ device implanted. Two were detected at one month, two at six months, and three at 12 months of follow up. The ratio of device diameter to the stretched diameter of the defect was similar in patients with and without fractures $(2.4(0.5) v 2.5(0.4)$ respectively, $\mathrm{p}=0.68)$. The fractures occurred at the joint level within the supporting arms and have not been associated with development of a residual leak or an increase in its size, if present. No device or device fragment embolisation has occurred. Protrusion of one arm of the umbrella through the defect was observed in $17 / 50$ patients $(34 \%)$, associated with the presence of deficient anterior $\operatorname{rim}(p<0.05)$. This finding did not affect the function of any cardiac structure; however, residual leaks were more commonly found in patients with protrusions $(\mathrm{p}<0.05)$. 


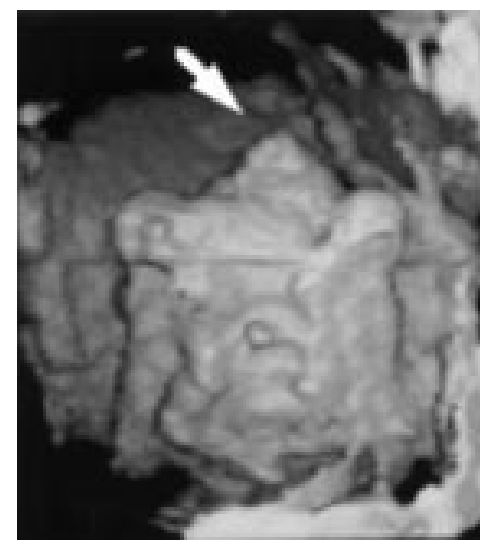

View from RA

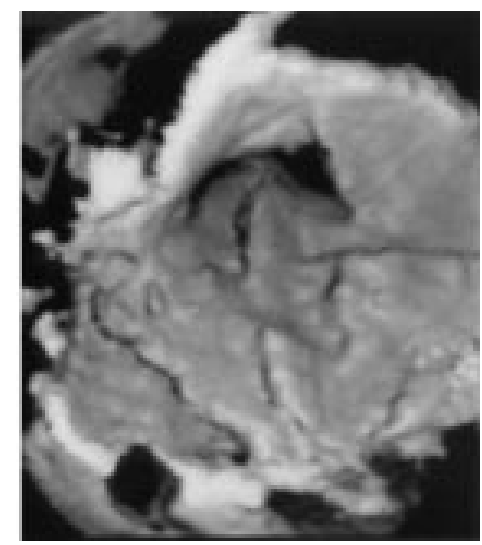

From LA
Figure 2 A three dimensional echocardiographic study after Cardio-Seal implantantion in the anatomical setting of a deficient anterosuperior rim: (left) (arrow) from the right atrial view; (right) from the left atrial view.

All patients were asymptomatic at the latest follow up. Although only $13 / 50$ patients $(26 \%)$ were symptomatic (fatigue) before the procedure, $31(62 \%)$ reported a subjective improvement in exercise tolerance and activity level after closure. Before the procedure, 45 patients $(90 \%)$ had clinical findings compatible with atrial septal defect on physical examination, two $(4 \%)$ were normal on examination (stroke patients), and three had clinical signs that were overshadowed by other associated cardiac defects. At the latest follow up, all patients with clinical findings of an atrial septal defect showed pronounced reduction or elimination of murmurs and normalisation of fixed splitting of the second heart sound. Forty six patients $(92 \%)$ had signs of a left to right shunt on chest $x$ ray before the procedure, and all showed pronounced reduction in cardiac size and pulmonary flow pattern. Two patients experienced palpitations that required investigation. Holter monitoring revealed short runs of atrial fibrillation in one and was normal in the other. One patient had a generalised seizure one year after the procedure. In this patient, magnetic resonance imaging of the brain was normal and TOE showed no residual atrial leak and no thrombus in the left atrium. One patient had a transient increase in known seizure activity immediately after the implantation, thought to be secondary to anaesthesia, and two complained of transient chest pain within the first month of follow up. Interestingly, five patients $(10 \%)$ experienced transient but significant headaches, three with migrainelike features; three of these five patients had a previous history of recurrent migraines or headaches, which worsened after implantation, and two had a family history of migraine or headaches. All patients had spontaneous improvement after six months of follow up. No patient has had any heart related hospital admissions, instances of late device migration, or episodes of endocarditis.

\section{Discussion}

In this report we document the acute and short term results of transcatheter closure of small to moderate sized atrial septal defects with the
Cardio-Seal device, performed in a single centre. The procedure was shown to be safe, with a low rate of immediate complications. The haemodynamic disturbances secondary to the right ventricular volume overload were corrected, with favourable clinical outcomes. Although the rate of residual shunting was $46 \%$ at follow up, septal motion abnormalities were eliminated in virtually all patients, and the right ventricular end diastolic dimension improved significantly after implantation. Not surprisingly, the reduction in right ventricular dimensions was less in patients with residual shunts than in those with complete occlusion, considering that the mean follow up was relatively short (9.9 months). ${ }^{9}$ On the basis of published data from the previous Clamshell trials, additional reduction in the size or resolution of residual shunts can be anticipated with time, leading to a further decrease in right heart dimensions. ${ }^{78}$ Right ventricular size may take 6-12 months to normalise, and sometimes may not return to normal even after many years following complete surgical closure at a young age. ${ }^{12-14}$ Nevertheless, our clinical and radiological findings support the view that haemodynamic improvement occurs despite the presence of small shunts. ${ }^{9}$

Septal motion and right ventricular dimensions more directly assess the haemodynamic effect of volume overload to the right ventricle. In this regard, measuring the dimensions of residual shunts with colour Doppler may not accurately reflect the burden to the right heart, and in some cases can be misleading, as the size or even the presence of a leak can be affected by turbulent flow patterns near the device and characteristics unique to patient imaging. ${ }^{9} 10$ This phenomenon was observed in four adults with residual leaks estimated at $>4 \mathrm{~mm}$ in diameter, despite their having normalised right ventricular dimension and septal wall motion. The remaining two patients with large colour Doppler shunts continue to have increased right ventricular end diastolic diameters and may be candidates for a second transcatheter procedure, or for surgical closure if these findings persist over longer follow up.

A recent multicentre trial using the same implant has shown that a stretched diameter of $>14 \mathrm{~mm}$ predisposes to moderate $(>2 \mathrm{~mm}$ ) residual leaks, although none has been clinically significant. ${ }^{15}$ In the present study we could not identify risk factors for residual shunting in the multivariate analysis. In the univariate analysis, however, defects with little or no rim, particularly in the anterosuperior portion of the septum, were associated with a greater incidence of residual leaking owing to arm protrusion. Three dimensional echocardiographic studies from this population have identified possible causes of such an event ${ }^{10}$ (fig 2). Underestimation of the longest dimension of an elliptical defect, by deforming its contour at the time of balloon sizing, and malalignment between the device and the plane of the atrial septum, accentuated by the tension on the delivery system at the time of deployment, may result in such positional anomalies. Additionally, acute angulation of the anterosuperior left 
atrial arm to the long axis of the ascending aorta when the anterior rim is deficient may also lead to arm protrusion after placement.

To reduce the likelihood of protrusion, the delivery system can be rotated while the proximal right atrial umbrella remains within the sheath, positioning the distal left atrial umbrella more parallel to the plane of the atrial septum or aorta. Release of the right atrial arms slightly off plane of the defect (more in the left atrium) can also help to avoid pulling the anterosuperior arm through a deficient rim (figs 1 and 2), allowing the anterosuperior arms to caress the roof of the left atrium behind the aorta. Before shaping the long sheath, making a slight curve at its end and directing it posteriorly in the left atrium may also be helpful, as can initial sheath placement into the right upper rather than the left upper pulmonary vein. We do not believe that the presence of a deficient anterosuperior rim should dissuade the interventionist from using this device. Indeed, the excellent haemodynamic success and low profile favour its use, even in this anatomical situation.

The ratio of device diameter to stretched diameter of the defect was more than $2: 1$ in this study (mean 2.5). In the first place, the Cardio-Seal device does not have a self centring mechanism, and in the worst scenario it is assumed that the device centre sits on the edge of the defect after implantation. Additionally, for patients with multiple atrial septal defects and defects with deficient anterior rims (of which there was a high incidence in this series), we deliberately chose larger devices to cover the entire defect and to optimise support around the defect. Finally, a ratio of 2.0 may not be appropriate for elliptical defects, which are difficult to assess on cross sectional TOE. ${ }^{10}$ The Starflex device (Nitinol Medical Technologies Inc, Boston, Massachusetts, USA), a design similar to the Cardio-Seal device but with a self centring mechanism, may address these issues, with lower device to stretched diameter ratios. Nevertheless, we did not have complications resulting from the use of slightly oversized devices.

Arm fractures have not been completely eliminated, although the incidence was significantly lower than with the Clamshell implant. ${ }^{78}$ Their presence has not resulted in an increased rate of residual shunting, device embolisation, or clinical complications, and the clinical significance (if any) of such fractures remains to be determined by longer follow up. On the basis of previous reports addressing the intermediate term outcomes (up to 10 years of follow up) with the Clamshell device, it is unlikely that such fractures will lead to any untoward events. $^{78}$

The potential risk of paradoxical emboli in patients with a residual atrial septal defect after transcatheter closure remains speculative. Prewitt and colleagues reported one patient who developed a transient ischaemic attack six months after a Clamshell occlusion in the setting of residual bidirectional shunt. ${ }^{16}$ Incomplete device endothelialisation was shown after device explantation and surgical repair. This is in contrast to previous animal studies that have shown complete endothelialisation three months after Clamshell implantations. ${ }^{17}$ As with the Clamshell device, the low profile of the Cardio-Seal device and the spring back mechanism are responsible for its good apposition to the atrial septal surface, facilitating endothelialisation and minimising atrial thrombus formation. Whether the presence of a small residual atrial communication exposes these patients to the risk of paradoxical emboli is not known. If a patent oval foramen functions only as a passage for a distally originating thrombus, this might indeed be the case. However, if the mechanism of cryptogenic stroke in the presence of a patent oval foramen is related to the flap valve of the septum primum itself, then the answer is perhaps not. Further, modifying influences affecting the endothelial cells lining the residual passage and the size and extent of such a shunt will need to be accounted for in the assessment of potential risk.

Electrophysiological studies in children before and after surgical closure of atrial septal defects suggest that the substrate for rhythm disturbances is present before surgery, predisposing them to future clinically significant arrhythmias. $^{18}$ The role of surgery in the genesis of these arrhythmias is unknown. Transcatheter closure of atrial septal defects has been shown to improve non-invasive electrophysiological findings at follow up. ${ }^{19}$ One may speculate that the avoidance of an atrial surgical scar should reduce the incidence of late supraventricular arrhythmias. The episode of atrial fibrillation in this series occurred in an adult with long standing right ventricular volume overload, and therefore it is unclear whether or not it was triggered by device implantation.

The potential advantages of transcatheter closure of an atrial septal defect over the surgical approach are obvious. They include the avoidance of a surgical scar, cardiopulmonary bypass, and a reduction in hospital stay. In addition, it has been suggested that there is a better neuropsychological outcome after device occlusion in children who undergo interventional versus surgical closure of a secundum atrial septal defect, although this was not found to be clinically significant. ${ }^{20}$ The relatively high incidence of significant but transient headaches after device closure in this series is an interesting observation of unknown aetiology. Perhaps such headaches are related to the release of vasoactive mediators from platelets during the early process of endothelialisation, as they tended to occur in families with migraine histories. Haemodynamic changes, such as an increased left atrial pressure with chamber distension, especially during exercise, may also play a role by causing release of vasoactive hormones.

CONCLUSIONS

In this study we report satisfactory short term clinical and haemodynamic results after transcatheter closure of small to moderate secundum atrial septal defects with the new CardioSeal device. Although these favourable results 
are similar to those achieved with the Clamshell device, our patients had a higher incidence of multiple defects and relatively deficient rims than reported previously. ${ }^{78}$ The problem of supporting arm fractures has not been completely eliminated, although the fracture rate has decreased substantially. No clinical complications have resulted from arm fracture, and from previous experience with the Clamshell device it is unlikely that any will occur in the future.

\section{Addendum}

After completion of this study, 50 additional patients have undergone transcatheter closure of a secundum atrial defect using a similar technique, with similar immediate outcomes. Nevertheless, a significant complication occurred during follow up that needs to be reported. A nine year old boy, weighing $32 \mathrm{~kg}$, had a $28 \mathrm{~mm}$ Cardio-Seal device implanted to close a central $9.5 \mathrm{~mm}$ (stretched diameter) defect with satisfactory rims. TOE showed good device position with no protrusion and no residual leak 20 minutes after the implantation. These findings were subsequently confirmed by transthoracic echocardiography the following morning and at the first month appointment. After six months, while playing in a park on holiday in another country, syncope occurred and the child was taken to the local hospital. Subsequent transthoracic echocardiography revealed a pericardial effusion with signs of tamponade and a retroaortic haematoma. After drainage and stabilisation, TOE showed that one arm of the device had perforated the roof of the left atrium behind the aorta. The patient was taken to theatre where the perforation was confirmed. The device was surgically explanted, the hole in the roof of the left atrium suture closed, and the defect patched with no complications. On microscopy the explanted device was almost fully endothelialised and the manufacturer found no abnormality in its structure after extensive assessment. After careful review of the postimplant echocardiographic studies, no anatomical or device related explanation for this occurrence could be found. No other cases of cardiac perforation secondary to Cardio-Seal implantation for the treatment of a variety of lesions, including atrial and muscular ventricular septal defects, have been reported so far. Likewise, no single case of perforation has been reported after more than a thousand Clamshell implantations. Pronounced thickening of the atrial wall adjacent to the device, with subsequent resolution, has been reported after Clamshell implantation. Progression of this type of lesion to the point of wall erosion and eventual perforation is, however, speculative, as no such lesion has been observed with the Cardio-Seal device. No focal thickening was observed on echocardiograms on this patient.

CACP was supported by a grant provided by the "Dante Pazzanese" Institute of Cardiology and the "Adib Jatene" Foundation, Sao Paulo, Brazil.

1 King TD, Thompson SL, Steiner C, et al. Secundum atrial septal defect: nonoperative closure during cardiac catheterization. $\mathscr{f A M A}$ 1976;235:2506-9.

2 Lock JE, Rome JJ, Davis R, et al. Transcatheter closure of atrial septal defects: experimental studies. Circulation 1989; 79:1091-9.

3 Rome JJ, Keane JF, Perry SB, et al. Double-umbrella closure of atrial septal defects: initial clinical applications. Circulation 1990;82:751-8.

4 Magee AG, Qureshi SA. Closure of atrial septal defects by transcatheter devices. Pediatr Cardiol 1997;18:326-7.

5 Bjornstad PG. The role of devices in the closure of atrial septal defects in the oval fossa. Cardiol Young 1998;8:2856.

6 Latson LA. The CardioSEAL device: history, techniques, results. F Intervent Cardiol 1998;11:501-5.

7 Prieto LR, Foreman CK, Cheatham JP, et al. Intermediateterm outcome of transcatheter secundum atrial septal defect closure using the Bard Clamshell Septal Umbrella. Am 7 Cardiol 1996;78:1310-12.

8 Justo RN, Nykanen DG, Boutin C, et al. Clinical impact of transcatheter closure of secundum atrial septal defects with

the double umbrella device. Am f Cardiol 1996;77;889-92. Boun C, Musewe NN, Smallhorn JF, et al. Echocardiographic follow-up of atrial septal defect after catheter 621-7.

10 Maeno YV, Benson LN, Boutin C. Impact of dynamic 3D ransesophageal echocardiography in the assessment of trial septal defects and occlusion by the double-umbrell device (CardioSEAL). Cardiol Young 1998;8:368-78.

11 Meyer RA. Pediatric echocardiography. Philadelphia: Lea \& Febiger, 1977:291-4.

12 Borow K, Karp R. Atrial septal defect: lessons from the past, directions for the future. N Engl f Med 1990;323:1698700 .

13 McNamara DG, Latson LA. Long-term follow-up of patients with malformations for which definitive surgical
repair has been available for 25 years or more. Am f Cardiol 1982;50:560-8

14 Meijboom F, Hess J, Szatmari A, et al. Long term follow-up (9 to 20 years) after surgical closure of atrial septal defect at a young age. Am $\mathcal{F}$ Cardiol 1993;72:1431-4.

15 Moore P, Benson LN, Berman W, et al. CardioSEAL ${ }^{\mathrm{TM}}$ device closure of secundum ASDs: how effective is it? Cirdevice closure of secundum A
culation 1998;98(suppl I):754.

16 Prewitt KC, Gaither NS, Farb A, et al. Transient ischemic attacks after long-term Clamshell occluder implantation for closure of atrial septal defects. Am Heart $\mathcal{F}$ 1992;124: for closure $1394-7$.

17 Kuhn MA, Latson LA, Cheatham JP, et al. Biological responses to Bard clamshell septal occluders in the canine heart. Circulation 1996;93:1459-63.

18 Bolens M, Friedli B. Sinus node function and conduction system before and after surgery for secundum atrial septal defect: an electrophysiology study. Am $\mathcal{F}$ Cardiol 1984;53: 1415-20.

19 Schenck MH, Sterba R, Foreman CK, et al. Improvement in noninvasive electrophysiologic findings in children after transcatheter atrial septal defect closure. Am f Cardiol 1995;76:695-8.

20 Visconti KJ, Bichell DP, Jonas RA, et al. Developmental outcome after surgical versus interventional closure of ASD2 in children [abstract]. Circulation 1998;98(suppl I):340. 